ISSN 1997-5902

\title{
Caractéristiques morpho-pédologiques et potentiels d'un sol de bas-fond secondaire développé sur granito-gneiss en région de savane guinéenne (Centre de la Côte d'Ivoire)
}

\author{
Akassimadou E.F. ${ }^{*}$ Yao-Kouamé A. ${ }^{1^{* *}}$ \\ 1 : Université Felix Houphouët-Boigny, Cocody, Abidjan. UFR Sciences de la terre et des ressources minières. \\ Département des sciences du sol (BP V34 Abidjan 22 Côte d'Ivoire) \\ *Auteur correspondant : email : akassdjaful@yahoo.fr ; Cel : +22507683230 \\ ${ }^{* *}$ Autre auteur : Email : yaokouamealbert1@yahoo.fr
}

Original submitted in on 24th February 2014. Published online at www.m.elewa.org on $31^{\text {st }}$ July 2014. http://dx.doi.org/10.4314/jab.v79i1.17

\begin{abstract}
RESUME
Objectif: Têtes des réseaux hydrographiques, les bas-fonds sont des milieux complexes, dont le fonctionnement physico-chimique est déterminé par les conditions hydriques. Ils sont souvent considérés comme des zones fertiles, bien que n'étant généralement pas suffisamment utilisés en Afrique de l'ouest. Aussi, pour mieux comprendre les phénomènes qui s'y déroulent et connaitre les bas-fonds en zone de savane guinéenne de Côte d'ivoire, une étude portant sur la caractérisation morpho-pédologique et physico-chimique des sols de bas-fond a-t-elle été initiée, en vue de déterminer les potentiels agronomiques réels de ces bas-fonds pour une agriculture durable dans cette agro-écologie.

Méthodologie et résultats : Un réseau de layons a été mis en place avec un layon de base parallèle à la direction générale du tronçon de bas-fond, sur $350 \mathrm{~m}$ couvrant le site de l'essai. A chaque $50 \mathrm{~m}$, un layon secondaire a été ouvert, perpendiculairement au tronçon entre les 2 versants hydromorphes du bas-fond. Sur les layons secondaires, à chaque $20 \mathrm{~m}$, la couleur du sol a été notée et la texture déterminée, pour les profondeurs $0-20 \mathrm{~cm}, 20-40 \mathrm{~cm}$ et $40-60 \mathrm{~cm}$ du sol. En outre un layon partant du sommet du paysage jusqu'au canal principal a été ouvert pour la réalisation d'une toposéquence comportant de 4 fosses (sommet, haut de versant ; bas-versant et bas-fond). Des échantillons de sol ont été prélevés dans chaque profil et un échantillon composite des sols du bas-fond a été constitué pour l'analyse chimique en laboratoire. Les résultats révèlent que $\mathrm{K}$ est l'élément nutritif déficient dans le sol, d'où la nécessité d'apporté du $\mathrm{K}$ si l'on veut accroitre les rendements agricoles de manière durable, la répartition des textures est équilibrée et symétrique par rapport au canal principal. On note également l'existence d'un recouvrement sableux (dans les parties latérales du bas-fond) sur des formations plus fines (argile et limon, dominantes dans la partie médiane), atténuant ainsi le potentiel agronomique et menaçant l'existence même de ce type de bas-fond dans la région.

Conclusion et application: Toutefois, ces sols présentent des textures équilibrées, permettant un bon drainage et empêchant ainsi des phénomènes de toxicité.
\end{abstract}

Mots clés : Bas-fond, potentiels, morpho-pédologique, recouvrement sableux, Côte d'Ivoire 
Morpho-pedological and potential features of lowland secondary soil developed on granito-gneiss in Guinean savanna region (Center of Côte d'Ivoire)

\section{Abstract}

Objectives: Heads of river systems or the Lowlands are the complex surroundings, whose physicochemical working is determined by the water conditions. They are often considered fertile zones, although not being fullly utilized in West Africa. To better understand the phenomena that take place and know the Lowlands in Guinea savanna zone of Côte d'Ivoire, a study of the morphological and soil physico-chemical characterization of soils was initiated in order to determine the actual agronomic potential of these Lowlands for sustainable agriculture in this agro-ecology.Methodology and Results: A transecting network was set up with a basic transect parallel to the general direction of the lowland section, $350 \mathrm{~m}$ covering the trial site. Every $50 \mathrm{~m}$, a secondary transect was opened perpendicular to the segment between the two sides of waterlogged lowland. On secondary transects, $20 \mathrm{~m}$ each, the ground color was noted and the texture determined for the depths $0-20 \mathrm{~cm}, 20-40 \mathrm{~cm}$ and $40-60 \mathrm{~cm}$ of the soil. In addition, a transect from the top of the landscape to the main channel has been opened for the construction of a toposequence having 4 pits (top, top side, bottom-side and low-background). Soil samples were collected from each profile and a composite sample of lowland soils was made for chemical laboratory analysis. The results show that $\mathrm{K}$ is deficient nutrient in the soil, which made the need for $\mathrm{K}$ if we want to increase agricultural productivity in a sustainable manner, the distribution of textures is balanced and symmetrical channel principal. It was also noted that the existence of a sandy cover (in the sides of the lowland) on (dominant in the middle part clay and silt) thinner formations, thus mitigating the agronomic potential and threatening the very existence of this type of lowland in the region. Conclusions and application of findings: However, these soils present balanced textures, permitting a good drainage and preventing phenomena of toxicity thus.

Key words : lowland, potential, morpho-pedological, sandy recovery, Côte d'Ivoire.

\section{INTRODUCTION}

Les définitions données au mot bas-fond diffèrent d'un auteur à un autre (Browers (1974); Killian et Teisser (1973); Ouedraogo (1987); Berton (1988), Zeppenfeld et Vlaar (1990); Jamin et Windmeijer (1996); Lidonetal. (1996); Raunet (1985) ; Delville et Boucher (1996)) ; ce qui traduit sa complexité à travers les aspects topographique, morphologique, pédologique et hydrologique. Toutefois, tous ces auteurs se rejoignent quant à l'importance des potentialités des bas-fonds dans le monde en général, et particulièrement en Afrique, où la sécheresse, la dégradation de certains sols et la pression démographique ont amené les paysans à chercher de nouvelles terres pour les cultures (Albergelet al ,1993). En Afrique subsaharienne les bas-fonds présentent un potentiel très important de développement et d'intensification de la production rizicole (Raunet, 1985 ; Mameriet al, 2008). La mise en valeur agricole de ces zones constituerait donc l'une des réponses possibles à la crise actuelle des systèmes traditionnels de production. Or, malgré leur surface estimée à 85 millions d'hectares, soit 7 p.c. De la superficie totale des terres cultivables, seulement 5 à 10 p.c. sont utilisées (Andriesse et Fresco, 1991; Lavigne et al, 1996). Ce désintéressement des bas-fonds est lié à plusieurs contraintes, notamment les problèmes d'aménagements(Lavigne et al, 1996),les conditions de travail, extrêmement difficiles dans les bas-fonds, mais surtout la méconnaissance des caractéristiques morpho-pédologiques et physico-chimiques des sols hydromorphes des bas-fonds, jouissant d'une particularité induite par la submersion (Ponnamperuma 1972; Vizier 1983).En effet, les bas-fonds sont des milieux complexes, fragiles, où peuvent apparaître de fortes contraintes hydrauliques et physicochimiques lors de la mise en culture (Lavigne et al, 1996). En outre, La valorisation de ces bas-fonds 
par les paysans, est le fruit de leurs stratégies de production, dans des contextes agro-écologiques et économiques donnés. Les pratiques paysannes de mise en valeur des bas-fonds s'inscrivent dans des systèmes de production diversifiés qui, sauf exception, sont centrés sur les cultures pluviales, répondant à des objectifs économiques qui ne passent pas forcément par l'intensification.

\section{MATÉRIELS ET MÉTHODE}

Zone d'étude : L'étude a été réalisée dans la vallée du M'be II ( $\left.8^{\circ} 06 \mathrm{~N}, 6^{\circ} 00 \mathrm{~W}, 180 \mathrm{~m}\right)$ au Centre de la Côte d'lvoire, dans un bas-fond semi-aménagé (barrage, canaux, casier non plané).C'est une zone de savane guinéenne située dans la zone de transition, entre la forêt et la zone de savane soudanienne plus au Nord (10e parallèle). Le régime pluviométrique y est bimodal, avec une température et une pluviométrie moyennes annuelles respectivement de $28^{\circ} \mathrm{C}$ et $1200 \mathrm{~mm}$. La roche-mère est majoritairement composée de granitogneiss et de granite calco-alcalin. Le site de l'expérimentation est un bas-fond irrigué semi aménagé drainé par un canal, occupé par une végétation secondaire, dominée par Lersiahexandra (poaceaes) et Fimbrinstylissp (poaceas) ainsi que des parcelles de riz de plusieurs variétés. Le paysage est constitué de plateaux à sommet cuirassé, démantelé, à versant rectiligne, convexe dans le bas de versant. Les sols de plateaux sont des ferralsols (Koné et al., 2009) alors que des fluvisols et gleysols ont été observés dans les bas-fonds de la zone par Diatta et Koné (2003).

Caractérisation morphologique du sol : Les sols du site d'expérimentation et de la zone ont fait l'objet d'une caractérisation morphologique et physico-chimique. Pour ce faire, un réseau de layons a été mis en place, avec un layon de base parallèle à la direction générale du tronçon de bas-fond, sur $350 \mathrm{~m}$, couvrant le site de l'essai. A chaque $50 \mathrm{~m}$, un layon secondaire a été ouvert, perpendiculairement au tronçon entre les 2 versants hydromorphes du bas-fond. Sur les layons secondaires, à chaque $20 \mathrm{~m}$, la couleur du sol a été notée à partir du code Munsell, et, la texture,

\section{RÉSULTATS}

Caractérisation morpho-pédologique et physicochimique des sols du site d'étude

Étude toposéquentielle : La toposéquence implantée comporte une fosse pédologique au sommet (plateau), une à mi versant, au bas versant (zone hydromorphe) et une dans le bas-fond. Les sols ont été décrits et
L'utilisation efficiente des bas-fonds commande alors une bonne connaissance de leurs caractéristiques morpho-pédologique et physicochimiques. C'est pourquoi, cette étude se propose de caractériser les sols d'un bas-fond secondaire sur granito-gneiss en région de savane guinéenne, en vue d'en déterminer les potentialités physicochimiques pour une production rizicole durable.

déterminée par la méthode tactile sur les profondeurs 0 $-20 \mathrm{~cm}, 20-40 \mathrm{~cm}$ et $40-60 \mathrm{~cm}$ du sol. En outre un layon partant du sommet du paysage jusqu'au canal principal a été ouvert pour l'implantation d'un topo séquence comportant plusieurs fosses. Quatre(4) fosses au total ont été ouvertes sur le layon depuis le sommet du paysage jusqu'au canal principal, à savoir, une fosse, au sommet, en haut de versant, au bas versant et dans le bas-fond. Des échantillons de sol ont été prélevés dans chaque profil du sol et un échantillon composite des sols du bas-fond a été constitué pour l'analyse chimique en laboratoire.

Analyses en laboratoire : Les échantillons composites du sol ont été séchés à l'air sous abri, puis passés au tamis $(2 \mathrm{~mm})$ avant d'être broyé. Le $\mathrm{pH}$ a été déterminé à l'électrode en verre dans un rapport de $1 / 2,5$ telle que décrite par Thomas (1996). La teneur du sol en Corganique a été déterminée par la méthode de Walkley et Black (Nelson et Sommers, 1982).Le phosphore total et le phosphore assimilable-Brayl, par la méthode décrite par Olsen et Sommers (1982). Les bases échangeables $(\mathrm{Ca}, \mathrm{Mg}$ et $\mathrm{K})$ ont été déterminées par l'extraction à l'acétate d'ammonium tamponné à $\mathrm{pH} 7,0$ avant la lecture au spectromètre à absorption atomiques $(\mathrm{Ca}, \mathrm{Mg})$ et à flamme $(\mathrm{K})$. L'azote total $(\mathrm{N}$ total) a été déterminé par la méthode de Kjeldahl (Bremner, 1996).

Analyse statistique: Une analyse de variance des données collectées a été réalisée à l'aide du logiciel SAS (StatisticalAnalysis System). Les tableaux et figures ont été élaborés à partir du tableur Microsoft Excel

classés selon les propriétés morphologiques (CPCS, 1967) comme l'indique la figure 1. L'occurrence d'un cuirassement à $110 \mathrm{~cm}$ de profondeur dans la fosse du sommet, et la présence de blocs de cuirasse isolés en surface témoignent d'un paysage de plateau cuirassé démantelé. En outre, à la limite de la zone 


\section{Akassimadou et Yao-Kouamé. J. Appl. Biosci. 2014. Caractéristiques morpho-pédologiques et potentiels d'un sol de bas-fond secondaire développe sur granito-gneiss en Cote d'Ivoire}

hydromorphe, il y'a un affleurement de cuirasse caractéristique d'un biseau cuirassé.

Typologie des sols et leur répartition le long de la toposéquence : La figure 1 montre la succession des types de sols du sommet vers le bas-fond, il s'agit de ferra sol de type ferralitique remanié, remanié induré ou appauvris sur le sommet et le mi-versant. Dans la zone hydromorphe, il s'agit de sol colluvionné hydromorphe de type aéronosol, avec occurrence d'une nappe perchée à environ $70 \mathrm{~cm}$ de profondeur. Des fluvisols caractérisent le bas-fond de façon dominante, à côté des gleysols minoritaires dans un environnement inondée. Le long de la toposéquence, on note une variation de la coloration des sols : rouge (2,5YR ; 5YR) jaune (7,5YR; 10YR), gris (10YR ; Gley) dans le basfond. De même, il y'a une tendance d'épaississement de la couche humifère.

Étude physico-chimique des sols des profils pédologiques: L'analyse physico-chimique des sols étudiés est présentée dans le tableau 1: On constate une différence texturale entre les sols du bas-fond et ceux du plateau (sommet, mi-versant et bas de versant). Sur le plateau, il y'a un enrichissement en sable, du sommet vers le bas de versant, alors que la teneur en argile est d'environ 39 p.c. dans le bas-fond. Les teneurs en carbone organique ( $\mathrm{C}$-org) et en azote total sont décroissantes le long de la toposéquence (du sommet vers le bas-fond). Les teneurs en $\mathrm{P}, \mathrm{Ca}, \mathrm{Mg}$ et $\mathrm{K}$, ainsi que la $\mathrm{CEC}$, présentent la même tendance d'évolution jusqu'au bas de versant (zone hydromorphe), avec, par contre, une augmentation dans le bas-fond. Dans ce paysage, les sols sont plus riches en sable, tandis qu'une déficience en $\mathrm{N}, \mathrm{P}$ et $\mathrm{Mg}$ s'observe dans l'horizon superficiel des sols de miversant et de bas de versant (zone hydromorphe). Une déficience en $\mathrm{K}\left(<0.10 \mathrm{cmolkg}^{-1}\right)$ est également notée dans cet horizon, au bas de versant (zone hydromorphe), avec une plus faible teneur en $C$ (0.19 $\left.\mathrm{gkg}^{-1}\right)$.

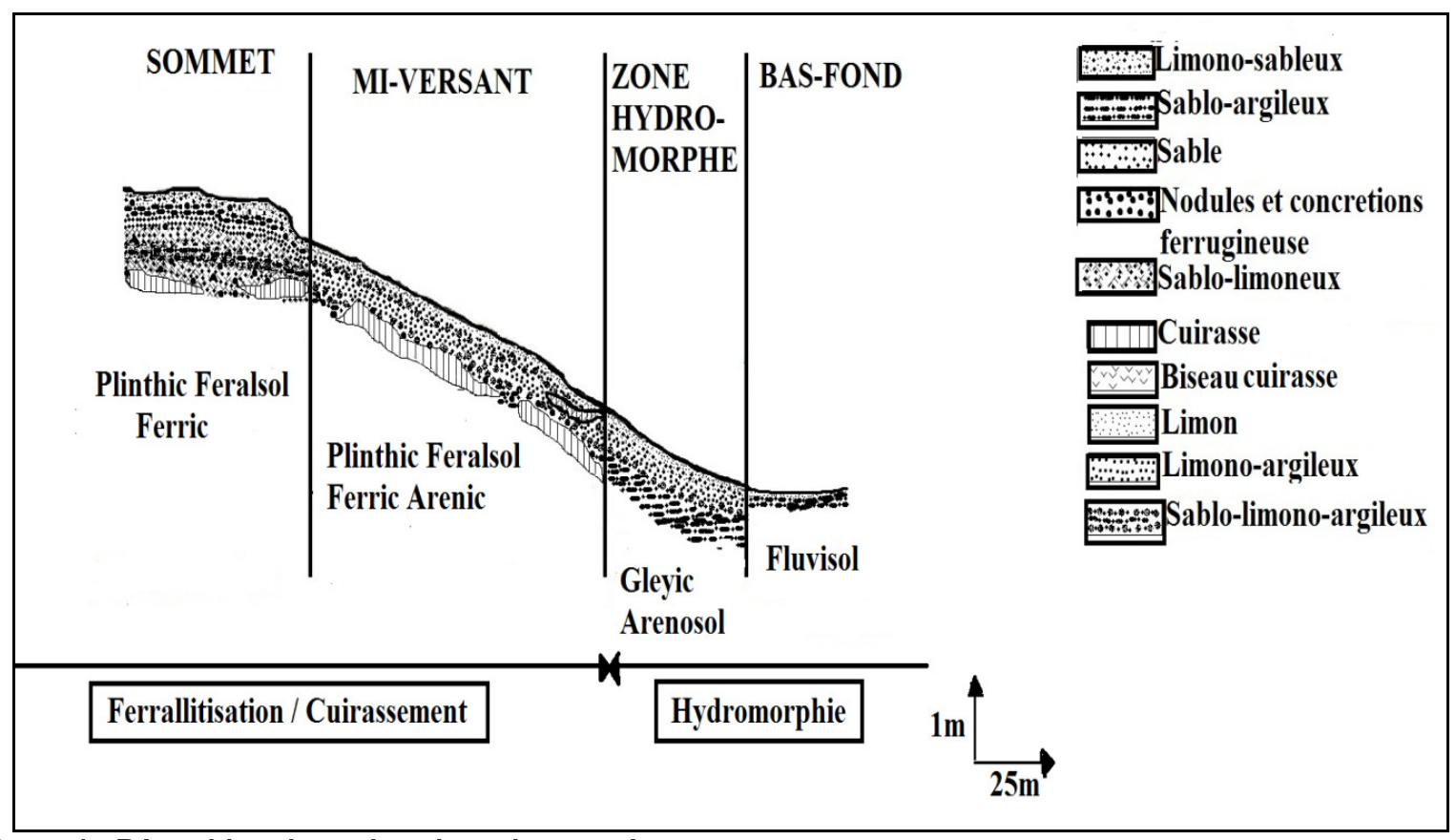

Figure 1 : Répartition des sols suivant la toposéquence 
Akassimadou et Yao-Kouamé. J. Appl. Biosci. 2014. Caractéristiques morpho-pédologiques et potentiels d'un sol de bas-fond secondaire développe sur granito-gneiss en Cote d'Ivoire

Tableau 1 : Analyse physico-chimique des sols le long de la toposéquence

\begin{tabular}{|c|c|c|c|c|c|c|c|c|c|c|c|c|}
\hline $\begin{array}{c}\text { Niveaux } \\
\text { observations }\end{array}$ & $\begin{array}{c}\mathrm{Hz} \\
(\mathrm{cm})\end{array}$ & $\begin{array}{c}\text { A } \\
(\text { p.c. })\end{array}$ & $\begin{array}{c}L \\
\text { (p.c.) }\end{array}$ & $\begin{array}{c}S \\
\text { (p.c.) }\end{array}$ & $\begin{array}{l}\text { C-o } \\
\text { (p.c.) }\end{array}$ & $\begin{array}{c}\mathrm{N}-\mathrm{t} \\
(\text { p.c. })\end{array}$ & $\begin{array}{l}\text { P-ass } \\
\text { (ppm) }\end{array}$ & $\begin{array}{c}\mathrm{Ca}^{2+} \\
(\mathrm{cmol} / \mathrm{kg})\end{array}$ & $\begin{array}{c}\mathrm{Mg}^{2+} \\
(\mathrm{cmol} / \mathrm{kg})\end{array}$ & $\begin{array}{c}\mathrm{K}^{+} \\
(\mathrm{cmol} / \mathrm{kg})\end{array}$ & $\begin{array}{c}\text { CEC } \\
\text { (cmol/kg) }\end{array}$ & $\begin{array}{c}V \\
\text { (p.c.) }\end{array}$ \\
\hline \multirow{5}{*}{ Sommet } & $0-22$ & 18 & 9 & 73 & 1,72 & 1,26 & 206 & 5,17 & 1,11 & 0,32 & 10,8 & 61,11 \\
\hline & $22-35$ & 40 & 13 & 47 & 0,98 & 0,73 & 163 & 3,30 & 0,74 & 0,18 & 8,2 & 47,92 \\
\hline & $35-60$ & 37 & 15 & 48 & 0,62 & 0,56 & 97,8 & 2,60 & 0,45 & 0,22 & 7,4 & 44,18 \\
\hline & $60-90$ & 53 & 12 & 35 & 0,45 & 0,45 & 54,3 & 2,40 & 0,41 & 0,22 & 8,6 & 35,23 \\
\hline & $90-110$ & 54 & 14 & 32 & 0,41 & 0,28 & 20,7 & 3,13 & 0,41 & 0,22 & 10,0 & 37,6 \\
\hline \multirow{5}{*}{ Mi- versant } & $0-10$ & 25 & 13 & 62 & 1,51 & 0,34 & 2,6 & 3,80 & 1,19 & 0,42 & 7,0 & 77,28 \\
\hline & $10-20$ & 33 & 14 & 53 & 0,83 & 0,67 & 0,43 & 2,30 & 0,86 & 0,10 & 5,8 & 56,20 \\
\hline & $20-30$ & 43 & 13 & 44 & 0,55 & 0,45 & 0,43 & 2,03 & 0,98 & 0,08 & 5,6 & 74,64 \\
\hline & $30-41$ & 53 & 12 & 35 & 0,48 & 0,56 & 0,43 & 1,75 & 1,09 & 0,08 & 7,8 & 37,43 \\
\hline & $41-70$ & 38 & 18 & 44 & 0,23 & 0,28 & 0,43 & 1,55 & 1,09 & 0,06 & 6,8 & 39,70 \\
\hline \multirow{2}{*}{ Zone Hydro } & $0-15$ & 10 & 7 & 83 & 0,19 & 0,34 & 3,0 & 0,95 & 0,36 & 0,08 & 2,0 & 69,5 \\
\hline & $35-50$ & 7 & 5 & 88 & 0,12 & 0,22 & 0,43 & 0,40 & 0,14 & 0,06 & 1,6 & 37,5 \\
\hline \multirow{4}{*}{ Bas-fond } & $0-20$ & 37 & 27 & 36 & 1,83 & 1,79 & 19,1 & 5,05 & 3,20 & 0,22 & 13,8 & 61,37 \\
\hline & $20-40$ & 36 & 21 & 43 & 0,40 & 0,45 & 1,70 & 3,50 & 2,94 & 0,08 & 9,6 & 67,91 \\
\hline & $40-60$ & 39 & 21 & 40 & 0,26 & 0,34 & 0,87 & 4,30 & 3,32 & 0,08 & 11,2 & 68,75 \\
\hline & $60-80$ & 37 & 24 & 29 & 0,24 & 0,34 & 0,87 & 4,40 & 3,60 & 0,10 & 11,4 & 71,05 \\
\hline
\end{tabular}

$\mathrm{Hz}$ :horizon; A :Argile ; L : Limon ; S :Sable ; C-o:Carbone Organique ; N-t : Azote total ; CEC :Capacité d'Échange Cationique ; V :Taux de saturation en base; Hydro : Hydromorphe 


\section{Akassimadou et Yao-Kouamé. J. Appl. Biosci. 2014. Caractéristiques morpho-pédologiques et potentiels d'un sol de bas-fond secondaire développe sur granito-gneiss en Cote d'Ivoire}

Étude texturale des sols du bas-fond : La répartition spatiale des textures du sol à différentes profondeur $(0$ $20 \mathrm{~cm} ; 20-40 \mathrm{~m} ; 40-60 \mathrm{~cm}$ ) est rapportée les figures 2,3 et 4 .On note une répartition presque symétrique des différentes textures par rapport à la zone médiane occupée par le canal, pour les trois niveaux de profondeurs. Sur les bords (figure 2), près du biseau cuirassé qui délimite la zone hydromorphe, on trouve des plages de sols sablo-argileux à sablo-limoneux, de l'amont vers l'aval. II y'a ensuite une incrustation de couche argilo-sableuse dans une plage argilo-limonosableuse excentrée, dans la partie Nord, tandis qu'au Sud, c'est une plage limono-argileuse qui est excentrée dans le bas-fond. En aval et dans la partie médiane, apparaissent une texture plus fine, de type argilolimoneux. Ensuite, cette texture est "encadrée » de part et d'autre, par des plages de sol respectivement argilo-sableux et sablo-limoneux. Par ailleurs une incrustation des sols sableux se rencontre à l'Ouest, vers l'aval, entre les textures argilo-sableuse et sablo- limoneuse. En effet, nous remarquons les mêmes textures (sableuse ; sablo-limoneuse ; argilo-sableuse et argilo-limono-sableuse), avec la même symétrie, et, plus ou moins, la même répartition dans l'espace. La synthèse de cette répartition spatiale des textures (figure 3) révèle qu'il y a seulement trois textures qui se rencontrent depuis la surface jusqu'à $60 \mathrm{~cm}$ de profondeur (la texture sablo-limoneuse, qui se rencontre exclusivement dans la zone hydromorphe, les textures argilo-sableuse et argilo-limono-sableuse, observée dans le bas-fond). La même symétrie apparait, avec des superpositions de textures allant des plus fines au plus grossières. De façon globale, l'on note une transgression des couches à texture grossière sur les textures plus fines, sur la coupe transversale du bas-fond. II se produit un rétrécissement de la largeur du bas-fond, au fur et à mesure qu'on remonte le layon vers l'amont. En effet, au départ, la largeur du bas-fond est 170 mètres environ, tandis qu'à $350 \mathrm{~m}$, sur le layon de base, la largeur n'est que $125 \mathrm{~m}$.

\section{A) Profondeur $0-20 \mathrm{~cm}$}

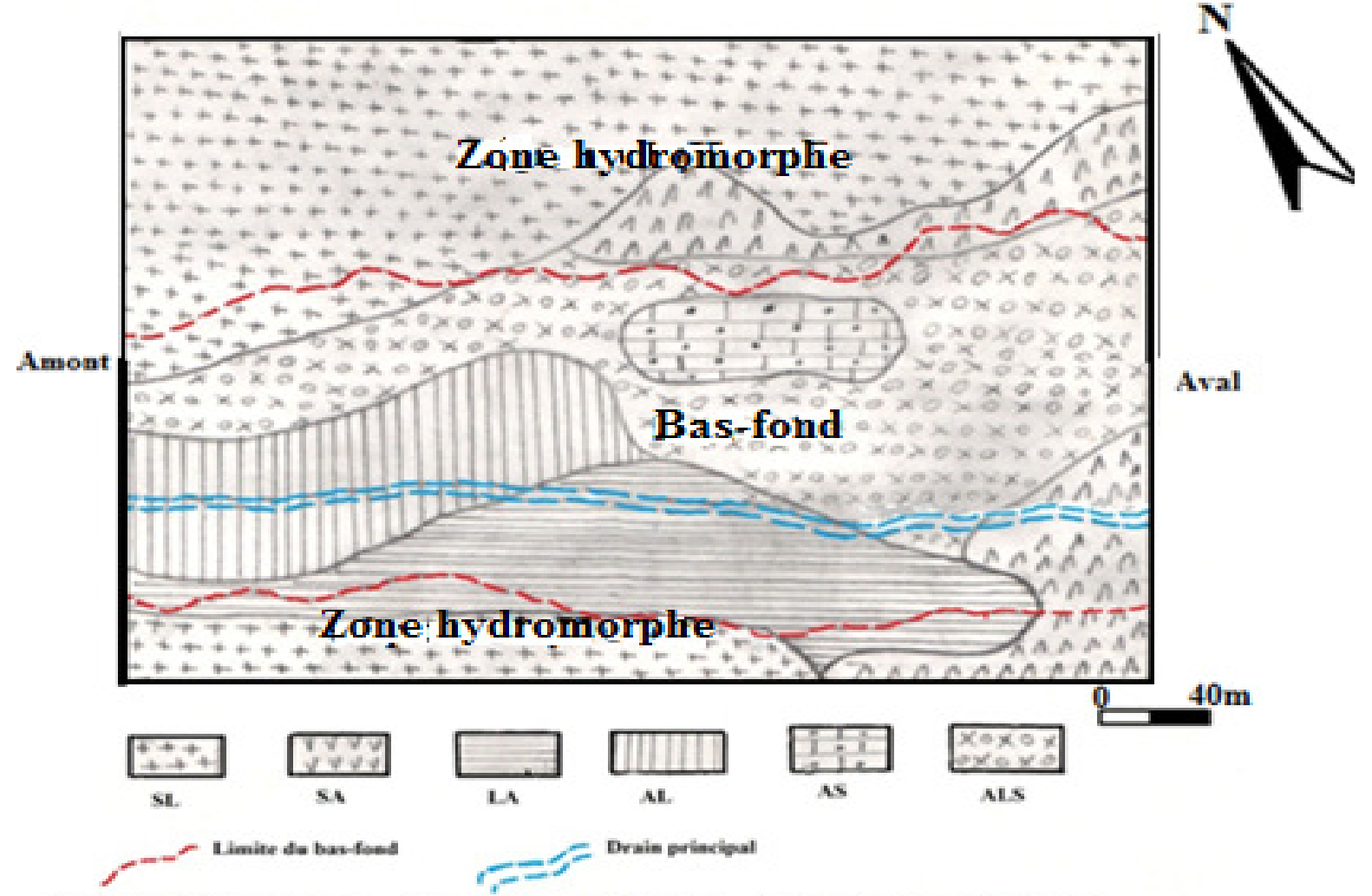

SL: Sablo-limoneuse; SA: Sablo-argileuse; AL: Argilo-limoneuse; AS: Argilo-sableuse;

LA: Limono-argileuse;

ALS: Argilo-limono-sableuse. 
Akassimadou et Yao-Kouamé. J. Appl. Biosci. 2014. Caractéristiques morpho-pédologiques et potentiels d'un sol de bas-fond secondaire développe sur granito-gneiss en Cote d'Ivoire

B) profondeur $20-40 \mathrm{~cm}$

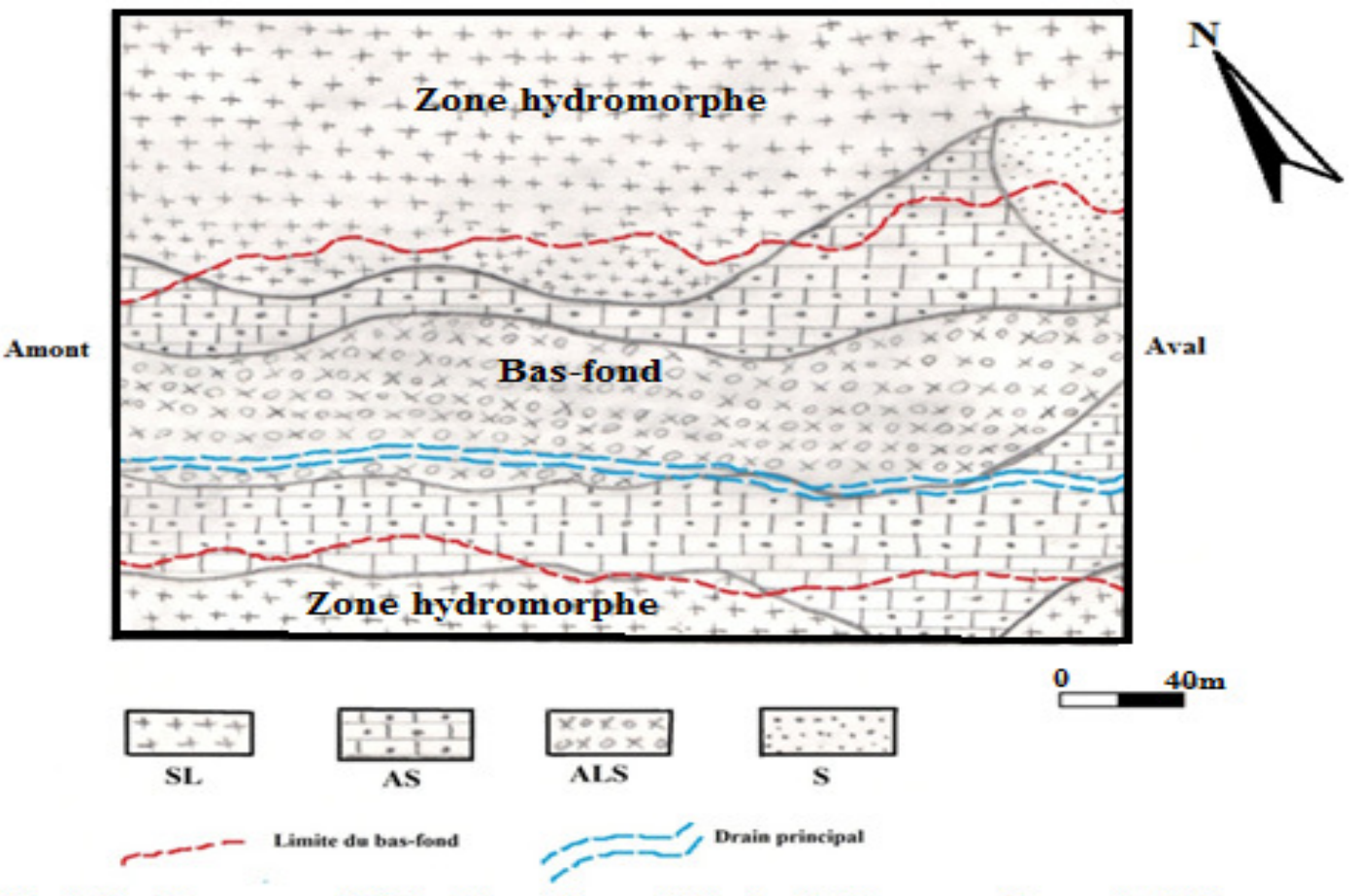

SL: Sablo-limoneuse ; AS: Argilo-sableuse; ALS: Argilo-limono-sableuse; S: Sableuse

C) Profondeur $40-60 \mathrm{~cm}$

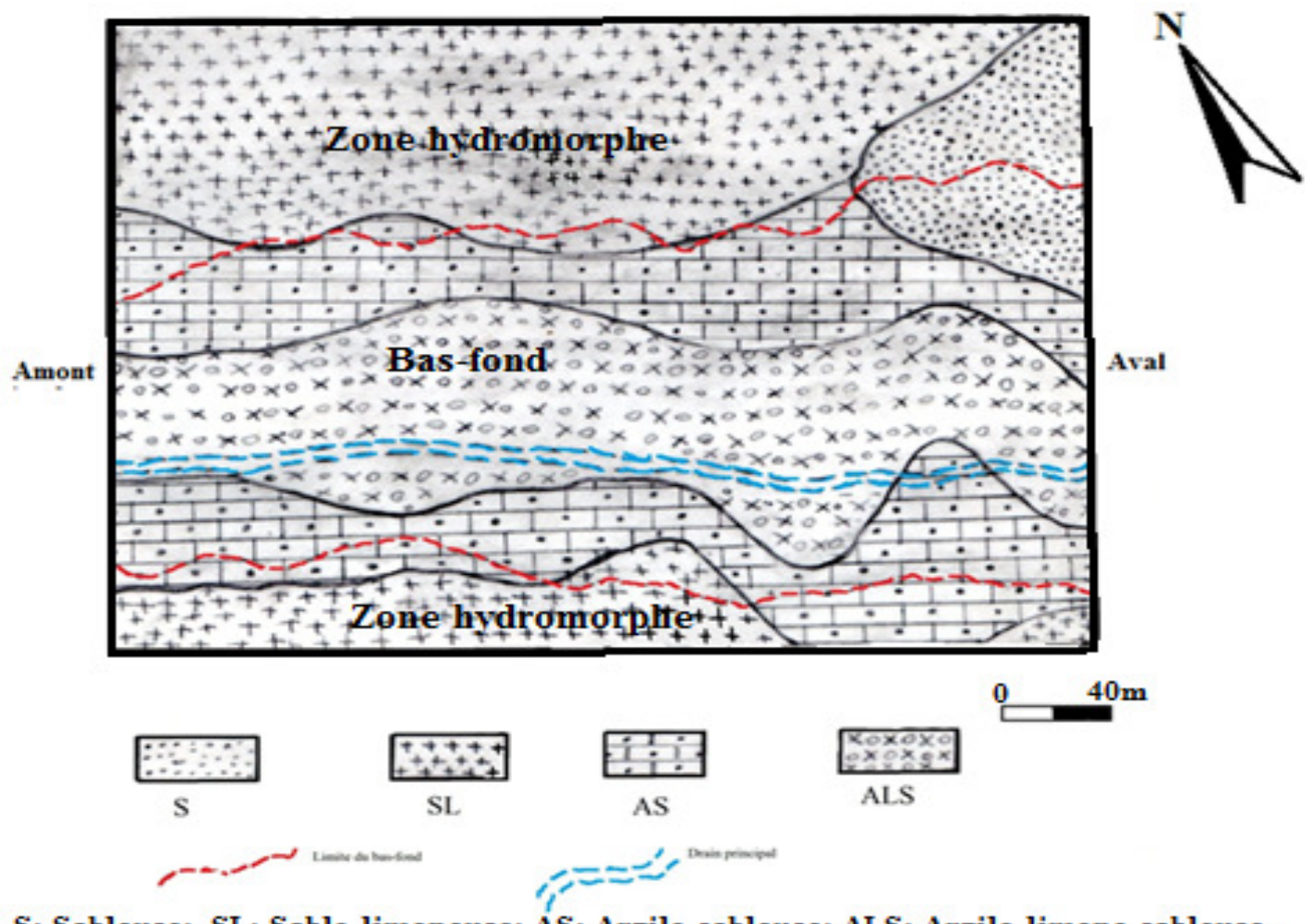

S: Sableuse; SL: Sablo-limoneuse; AS: Argilo-sableuse; ALS: Argilo-limono-sableuse.

Figure 2 : Carte des répartitions des textures selon les profondeurs $(A: 0-20 \mathrm{~cm}, B: 20-40 \mathrm{~cm} C: 40-60 \mathrm{~cm})$ 

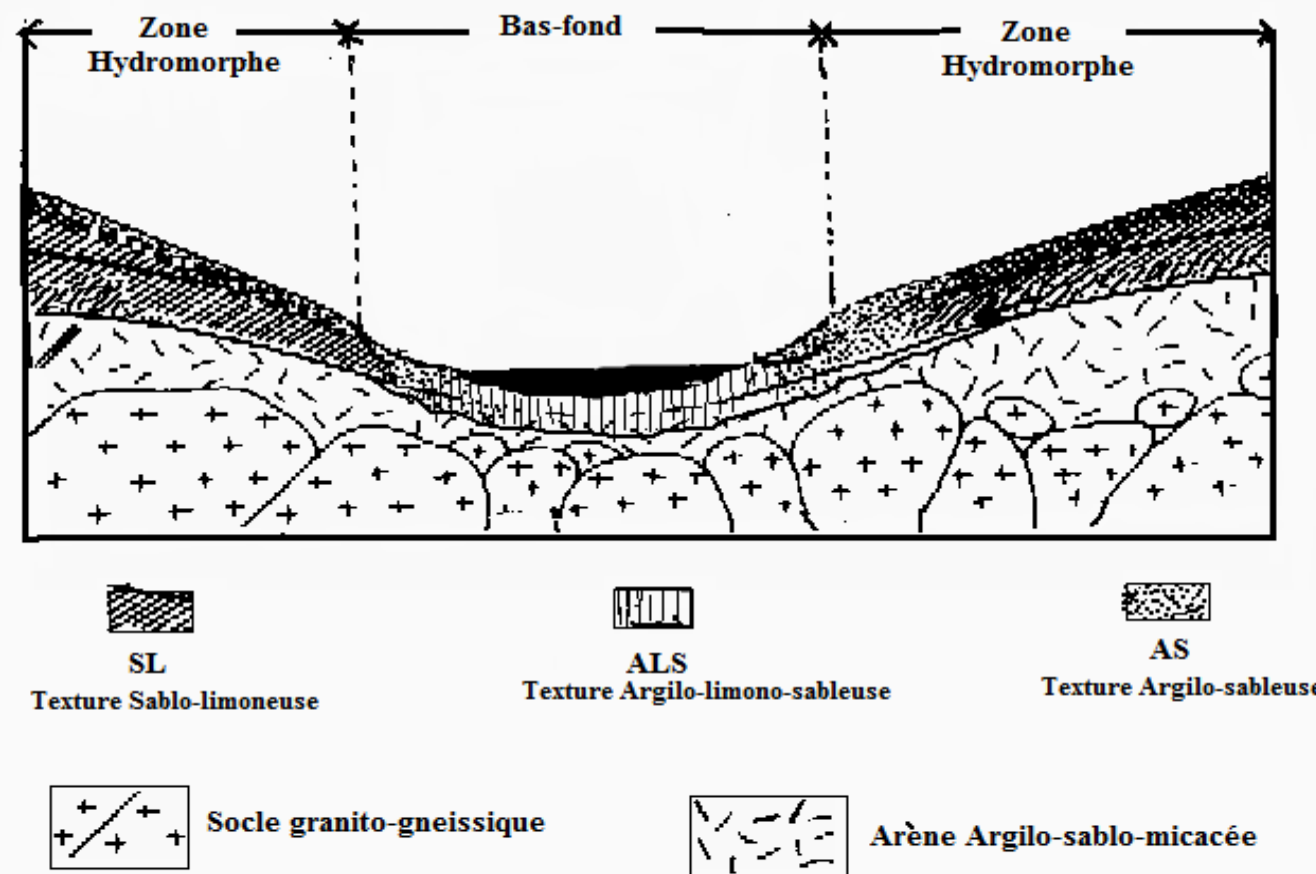

Arène Argilo-sablo-micacée

NB: Par souci de clarté, les épaisseurs relatives des divers matériaux, les dimensions des modélés, les pentes et les dénivellations n'ont pas êté respectées.

Figure 3 : Coupe transversale de la synthèse de la texture du sol

Variation de la couleur du sol du bas-fond

Évolution longitudinale et transversale des teintes selon les profondeurs : La distribution des fréquences relatives de la couleur du sol dans les différentes couches P1 $(0-20 \mathrm{~cm})$, P2 $(20-40 \mathrm{~cm})$ et P3 $(40-60 \mathrm{~cm})$, le long des du layon de base est présentée dans le tableau 2. Cette répartition a été significative avec une probabilisé $X^{2}$ de 0,019 dans la couche P2 $(20-40 \mathrm{~cm})$, alors qu'elle ne l'est pas pour les autres profondeurs P1 $(0-20 \mathrm{~cm})$ et P3 $(40-$ $60 \mathrm{~cm})$. On observe notamment, une croissance des fréquences des teintes $5 Y$ et $7,5 Y R$ ainsi qu'une une réduction de celle de 2,5Y de l'aval $(0 \mathrm{~m})$ vers l'amont $(350 \mathrm{~m})$, pour les trois profondeurs. Aucune tendance nette n'a été observée pour les autres teintes du sol. Le tableau 3 présente la synthèse de l'évolution transversale des teintes selon la profondeur, à la fois dans la zone hydromorphe et dans le bas-fond. L'analyse de ce tableau révèle que la distribution des teintes est très hautement significative $\left(X^{2}<0,001\right)$ pour les profondeurs $P 1$ et $P 3$, et hautement significative $\left(X^{2}=0,0002\right)$ pour la profondeur $P 2$. Les teintes $2,5 Y, 5 Y$ et $10 Y R$ sont fréquentes, à la fois dans le basfond et dans la zone hydromorphe. En revanche, la teinte 7,5YR apparait, préférentiellement, dans la zone hydromorphe, tandis que la Gley1 se trouve exclusivement dans le bas-fond. On note également une croissance de fréquences de la teinte $2,5 \mathrm{Y}$, de $\mathrm{P} 1$ à $\mathrm{P} 3$, dans le bas-fond et dans la zone hydromorphe, alors que la teinte $5 \mathrm{Y}$ décroit de la profondeur de P1 à P3. 
Akassimadou et Yao-Kouamé. J. Appl. Biosci. 2014. Caractéristiques morpho-pédologiques et potentiels d'un sol de bas-fond secondaire développe sur granito-gneiss en Cote d'Ivoire

Tableau 2 : Évolution longitudinale (layon de base) de la teinte du sol selon la profondeur du sol

Fréquence (p.c.) le long du layon de base

\begin{tabular}{|c|c|c|c|c|c|c|c|c|c|c|}
\hline \multirow[b]{2}{*}{ Teinte } & \multirow[b]{2}{*}{ prof } & & & & & & & & & \multirow[b]{2}{*}{$\mathrm{X}^{2}$-Prob } \\
\hline & & $0 \mathrm{~m}$ & $50 \mathrm{~m}$ & $100 \mathrm{~m}$ & $150 \mathrm{~m}$ & $200 \mathrm{~m}$ & $250 \mathrm{~m}$ & $300 \mathrm{~m}$ & $350 \mathrm{~m}$ & \\
\hline \multirow{3}{*}{$2,5 Y$} & $\mathrm{P} 1$ & 64,29 & 16,67 & 7,69 & 25,00 & 18,18 & 9,09 & 16,67 & 8,33 & 0,0809 \\
\hline & $\mathrm{P} 2$ & 57,14 & 41,67 & 7,69 & 0,00 & 36,36 & 36,36 & 25,00 & 16,67 & 0,0191 \\
\hline & P3 & 53,85 & 41,67 & 53,85 & 50,00 & 36,36 & 36,36 & 44,45 & 25,00 & 0,7685 \\
\hline \multirow{3}{*}{$5 Y$} & $\mathrm{P} 1$ & 28,57 & 58,33 & 61,54 & 50,00 & 63,64 & 45,45 & 41,67 & 50,00 & 0,0809 \\
\hline & $\mathrm{P} 2$ & 0,00 & 16,67 & 30,77 & 45,45 & 54,55 & 45,45 & 33,33 & 25,00 & 0,0191 \\
\hline & P3 & 15,38 & 16,67 & 0,00 & 25,00 & 36,36 & 27,27 & 18,118 & 25,00 & 0,7685 \\
\hline \multirow{3}{*}{ 7,5YR } & $\mathrm{P} 1$ & 7,14 & 0,00 & 7,69 & 25,00 & 9,09 & 18,18 & 0,00 & 16,67 & 0,0809 \\
\hline & $\mathrm{P} 2$ & 7,14 & 0,00 & 15,38 & 18,18 & 0,00 & 18,18 & 16,67 & 16,67 & 0,0191 \\
\hline & P3 & 0,00 & 8,33 & 23,08 & 16,67 & 18,18 & 27,27 & 18,18 & 25,00 & 0,7685 \\
\hline \multirow{3}{*}{ 10YR } & $\mathrm{P} 1$ & 0,00 & 16,67 & 7,69 & 0,00 & 9,09 & 27,27 & 33,33 & 16,67 & 0,0809 \\
\hline & $\mathrm{P} 2$ & 35,71 & 41,67 & 23,08 & 36,36 & 9,09 & 0,00 & 25,00 & 33,33 & 0,0191 \\
\hline & P3 & 30,77 & 33,33 & 23,08 & 8,33 & 9,09 & 9,09 & 18,18 & 16,67 & 0,7685 \\
\hline \multirow{3}{*}{ Gley 1} & P1 & 0,00 & 8,33 & 15,38 & 0,00 & 0,00 & 0,00 & 8,33 & 8,33 & 0,0809 \\
\hline & $\mathrm{P} 2$ & 0,00 & 0,00 & 23,08 & 0,00 & 0,00 & 0,00 & 0,00 & 8,33 & 0,0191 \\
\hline & P3 & 0,00 & 0,00 & 0,00 & 0,00 & 0,00 & 0,00 & 0,00 & 8,33 & 0,7685 \\
\hline
\end{tabular}

P1 (0-20 cm), P2 $(20-40 \mathrm{~cm})$ et P3 $(40-60 \mathrm{~cm})$ 
Akassimadou et Yao-Kouamé. J. Appl. Biosci. 2014. Caractéristiques morpho-pédologiques et potentiels d'un sol de bas-fond secondaire développe sur granito-gneiss en Cote d'Ivoire

Tableau 3 : Évolution transversale (distance) de la teinte du sol selon la profondeur du sol

Fréquence (p.c.) le long des layons secondaires

$\begin{array}{lllllllllllllll}\text { teinte prof } & 0 \mathrm{~m} & 20 \mathrm{~m} & 40 \mathrm{~m} & 60 \mathrm{~m} & 80 \mathrm{~m} & 100 \mathrm{~m} & 120 \mathrm{~m} & 140 \mathrm{~m} & 160 \mathrm{~m} & 180 \mathrm{~m} & 200 \mathrm{~m} & 220 \mathrm{~m} & 240 \mathrm{~m} & 260 \mathrm{~m}\end{array}$

\begin{tabular}{|c|c|c|c|c|c|c|c|c|c|c|c|c|c|c|c|}
\hline & P1 & 12,5 & 37,5 & 12,5 & 25 & 12,5 & 12,5 & 14,29 & 25 & 00 & 50 & 25 & 16,67 & 33,33 & 100 \\
\hline \multirow[t]{3}{*}{$2,5 \mathrm{Y}$} & P2 & 25 & 00 & 25 & 12,5 & 37,5 & 25 & 28,57 & 37,5 & 75 & 12,5 & 25 & 40 & 33,33 & 00 \\
\hline & P3 & 14,29 & 25 & 62,5 & 75 & 25 & 37,5 & 57,14 & 75 & 87,15 & 37,5 & 12,5 & 00 & 00 & 00 \\
\hline & P1 & 25 & 37,5 & 62,5 & 75 & 50 & 75 & 85,71 & 75 & 100 & 12,5 & 00 & 00 & 33,33 & 00 \\
\hline \multirow[t]{3}{*}{$5 Y$} & P2 & 12,5 & 62,5 & 62,5 & 37,5 & 25 & 62,5 & 57,14 & 37,5 & 12,5 & 00 & 00 & 00 & 00 & 00 \\
\hline & P3 & 28,57 & 12,5 & 25 & 12,5 & 62,5 & 37,5 & 42,86 & 12,5 & 12,5 & 00 & 00 & 00 & 00 & 00 \\
\hline & P1 & 37,5 & 00 & 00 & 00 & 00 & 00 & 00 & 00 & 00 & 00 & 50 & 50 & 00 & 00 \\
\hline \multirow[t]{3}{*}{ 7,5YR } & P2 & 62,5 & 00 & 00 & 00 & 00 & 00 & 00 & 00 & 00 & 12,5 & 37,5 & 40 & 00 & 00 \\
\hline & P3 & 57,14 & 00 & 12,5 & 00 & 00 & 00 & 00 & 00 & 00 & 12,5 & 62,5 & 60 & 66,67 & 00 \\
\hline & P1 & 25 & 25 & 12,5 & 00 & 00 & 00 & 00 & 00 & 00 & 37,5 & 25 & 33,33 & 33,33 & 00 \\
\hline \multirow[t]{3}{*}{ 10YR } & P2 & 00 & 37,5 & 00 & & & 12,5 & 14,29 & 25 & 12,5 & 75 & 37,5 & 20 & 66,67 & 100 \\
\hline & P3 & 00 & 62,5 & 00 & 12,5 & 12,5 & 25 & 00 & 00 & 00 & 50 & 25 & 40 & 33,33 & 00 \\
\hline & P1 & 00 & 00 & 12,5 & 00 & & 12,5 & 00 & 00 & 00 & 00 & 00 & 00 & 00 & 00 \\
\hline \multirow[t]{3}{*}{ Gley1 } & P2 & 00 & 00 & 12,5 & 25 & 12,5 & 00 & 00 & 00 & 00 & 00 & 00 & 00 & 00 & 00 \\
\hline & P3 & 00 & 00 & 00 & 00 & 00 & 00 & 00 & 12,5 & 00 & 00 & 00 & 00 & 00 & 00 \\
\hline & & & & & P1 & & & P2 & & & P3 & & & & \\
\hline \multicolumn{3}{|c|}{ Probabilité $\quad X^{2}$} & & & $<0,001$ & & & 0,0002 & & & $<0,001$ & & & & \\
\hline \multicolumn{3}{|c|}{ Degré de liberté } & & & 52 & & & 52 & & & 52 & & & & \\
\hline \multicolumn{3}{|c|}{ Nombre d'échantillons } & & & 97 & & & 96 & & & 95 & & & & \\
\hline
\end{tabular}

P1 $(0-20 \mathrm{~cm}), \mathrm{P} 2(20-40 \mathrm{~cm})$ et P3 $(40-60 \mathrm{~cm})$ 


\section{Akassimadou et Yao-Kouamé. J. Appl. Biosci. 2014. Caractéristiques morpho-pédologiques et potentiels d'un sol de bas-fond secondaire développe sur granito-gneiss en Cote d'Ivoire}

Propriétés physico-chimiques des sols du bas-fond Composition granulométrique: Le tableau 4 présente la proportion moyenne de la fraction granulométrique (argile, limon fin, limon grossier, sable fin et sable grossier) du sol du site d'étude. On note que le sol du bas-fond étudié est riche en argile, représentant environ 38 p.c. de la composition granulométrique du sol. La teneur de limon fin (Lf) est environ deux fois plus élevée que celui du limon grossier $(\mathrm{Lg})$, tandis que le sable fin (Sf) ne constitue que le tiers du sable total. En outre, le pourcentage de limon est sensiblement égal à celui du sable, avec, respectivement, 31,23 p.c. et 30,5 p.c. confirmant ainsi la texture argilo-limono-sableuse dominante au niveau du bas-fond.

Composition chimique : L'analyse de la composition chimique du sol du bas-fond (tableau 5), montre qu'il s'agit d'un sol moyennement acide $(\mathrm{pH}: 5,5)$ et riche en matière organique. II a une teneur très faible en azote et est déficient en potassium $\left(0,08 \mathrm{cmol}^{-\mathrm{kg}^{-1}}\right)$. Ce sol contient des teneurs normales en calcium

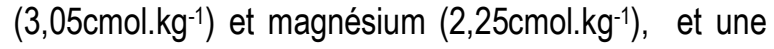
capacité d'échange cationique (CEC) élevée $\left(20,2 \mathrm{cmol} \mathrm{kg}^{-1}\right)$. Les résultats de l'analyse chimique révèlent également que ce sol est très riche en phosphore comme en témoigne la teneur en $\mathrm{P}$ assimilable (150ppm). Dans ce sol, on remarque que le complexe d'échange est fortement desaturé $(V=26,68)$ en surface $(0-20 \mathrm{~cm})$. Le rapport $\mathrm{C} / \mathrm{N}$ est égal à 10,2 dans le sol de bas-fonds.

Équilibres minéraux: Les différents équilibres minéraux considérés dans cette étude sont: $\mathrm{Nt} / \mathrm{P}_{2} \mathrm{O}_{5}$ total, $\mathrm{Ca} / \mathrm{Mg}, \mathrm{Mg} / \mathrm{K}, \mathrm{Ca} / \mathrm{K}, \mathrm{K} /(\mathrm{Ca}+\mathrm{Mg})$ et $\mathrm{K} / \mathrm{CEC}$. Les résultats de ces différents équilibres sont consignés dans le tableau 6. La valeur du rapport $\mathrm{Ca} / \mathrm{Mg}$ est faible $(\mathrm{Ca} / \mathrm{Mg}<1,5)$. Ce résultat suggère qu'il y a une légère déficience du calcium sur le magnésium dans le sol. $\mathrm{Ce}$ tableau révèle également que les valeurs du rapport $\mathrm{Mg} / \mathrm{K}$ et $\mathrm{Ca} / \mathrm{K}$ sont très élevées $(\mathrm{Mg} / \mathrm{K}>4$ et $\mathrm{Ca} / \mathrm{K}>$ 12). II résulte de ce qui précède que le magnésium et le calcium sont en excès par rapport au potassium dans ces sols.

Par ailleurs, les valeurs des rapports $\mathrm{K} /(\mathrm{Ca}+\mathrm{Mg})$ et $\mathrm{K} / \mathrm{CEC}$ sont très faibles $((\mathrm{K} /(\mathrm{Ca}+\mathrm{Mg})<2$ et $\mathrm{K} / \mathrm{CEC}<2)$ dans le sol de bas-fond. Le potassium est déficient par rapport à la somme du calcium et du magnésium sur le complexe adsorbant du sol.

Tableau 4: Composition granulométrique du sol du bas-fond

\begin{tabular}{ll}
\hline Fractions granulométriques & $\mathbf{0 - 2 0} \mathbf{~ c m}$ \\
\hline Argile (p.c.) & 37,45 \\
Limon fin (Lf) (p.c.) & 22,025 \\
Limon grossier (Lg) (p.c.) & 9,21 \\
Sable fin (Sf) (p.c.) & 10,24 \\
Sable grossier (Sg) (p.c.) & 20,26 \\
\hline
\end{tabular}

Tableau 5 : Caractéristiques chimiques du sol du bas-fond

\begin{tabular}{|c|c|}
\hline paramètres & $0-20 \mathrm{~cm}$ \\
\hline pHeau & 5,5 \\
\hline Matière organique $\mathrm{C}\left(\mathrm{g} \mathrm{kg}^{-1}\right)$ & 31,2 \\
\hline$N-t\left(g ~ k g^{-1}\right)$ & 3,1 \\
\hline $\mathrm{C} / \mathrm{N}$ & 10,06 \\
\hline Ptotal (ppm) & 365 \\
\hline Pass (ppm) & 150 \\
\hline CEC $\left(\mathrm{cmol} \mathrm{kg}^{-1}\right)$ & 20,2 \\
\hline $\mathrm{Ca}^{2+}\left(\mathrm{cmolkg}^{-1}\right)$ & 3,05 \\
\hline $\mathrm{Mg}^{2+}\left(\mathrm{cmol} \mathrm{kg}^{-1}\right)$ & 2,26 \\
\hline $\mathrm{K}^{+}\left(\mathrm{cmol} \mathrm{kg}^{-1}\right)$ & 0,08 \\
\hline$V($ p.c. $)$ & 26,68 \\
\hline
\end{tabular}


Tableau 6 : Équilibres entre minéraux du sol de bas-fond

\begin{tabular}{ll}
\hline paramètres & $\mathbf{0 - 2 0} \mathbf{~ m}$ \\
\hline $\mathrm{Nt} / \mathrm{Pt}$ & 8,49 \\
$\mathrm{Ca} / \mathrm{Mg}$ & 1,35 \\
$\mathrm{Mg} / \mathrm{K}$ & 28,25 \\
$\mathrm{Ca} / \mathrm{K}$ & 38,12 \\
$\mathrm{~K} /(\mathrm{Ca}+\mathrm{Mg})$ & 0,015 \\
$\mathrm{~K} / \mathrm{CEC}$ & 0,004 \\
\hline
\end{tabular}

\section{DISCUSSION}

L'étude morpho-pédologique du site étudié montre l'existence d'un recouvrement sableux sur des formations plus fines (argile et limon), dominantes dans la partie médiane du bas-fond. Ce recouvrement indique une certaine transgression de couches moins fertiles sur celles plus fertiles, dans la partie latérale, attestant d'un colluvionnement récent. Cette situation serait, d'une part, la conséquence de la dynamique de l'eau le long de la toposéquence, marquée par l'érosion des sols de plateau, dont les particules plus fines (argile et limon) sont transportées et déposées plus loin, dans le bas-fond, alors que les plus grosses sont présentes sur les bordures. A terme, ce processus géodynamique pourrait conduire à un élargissement de la zone hydromorphe, au détriment du bas-fond proprement dit, rejoignant en cela, Raunet (1985), qui avait signalé ce fait lors de son étude des bas-fonds de l'Afrique de l'Ouest, ou les travaux de Razafindrakoto (2007) effectués à Madagascar. Cette situation pourrait être aussi la conséquence des phénomènes d'hydromorphie. En effet, le bas-fond est soumis à des alternances de phases de submersion et d'exondation, qui déterminent son fonctionnement physico-chimique. Ainsi donc, temporaire ou permanente, l'hydromorphie joue donc un rôle déterminant : les sols de bas-fonds sont soumis à un intense lessivage, vertical ou longitudinal, souvent à l'origine de l'individualisation, sous l'horizon de surface, d'horizons de sables ou d'argiles blanches, totalement lessivés. Ces flux internes peuvent également emporter les particules fines, enrichissant en sables les zones amont ou latérales des bas-fonds. Lavigne et al, (1996) ont observé le même phénomène d'hydromorphie en Afrique subsaharienne humide. Notre étude révèle également que le processus de dégradation des basfonds est prononcé dans le bas-fond secondaire étudié, à travers l'existence d'une poche sableuse jusqu'à la partie médiane, réduisant du coup, le potentiel agronomique, et menaçant l'existence de ce type de bas-fond. Les sols des autres segments topographiques, notamment, au niveau du bas de versant, présentent plusieurs déficiences en nutriments pour les cultures vivrières, à l'exception du bas-fond. Cette analyse soulève la question de gestion rationnelle des ressources en sol, ce qui justifie l'exclusion des sols de bas de versant pour une culture vivrière intensive (Koné et al. 2009). Toutefois, l'existence d'une nappe phréatique au niveau du sol $(>70 \mathrm{~cm})$ de bas de versant lui confère, temporairement, des propriétés favorables à des cultures spécifiques (riz pluvial, maraîcher.) Dans le bas-fond proprement dit, on a noté une texture équilibrée, qui pourrait se prêter à une bonne riziculture irriguée. En effet, du point de vue aptitude rizicole ces sols relativement plus fertiles que ceux de la zone hydromorphe (Koné et al 2008)à cause de l'accroissement de la couche humifère dénotant ainsi d'une faible minéralisation de la matière organique due à la nappe qui crée un environnement inondé. Ce qui rend ce milieu peu pourvu en azote comme l'atteste le faible taux de carbone et d'azote obtenus par les analyses chimiques. Cependant, il pourrait y avoir une déficience en $\mathrm{K}\left(<0,10 \mathrm{cmolkg}^{-1}\right)$ dans la couche 20 $60 \mathrm{~cm}$ de profondeur du sol, qui va affecter le rendement de cette culture. Le $\mathrm{K}$ pourrait donc constituer le facteur déterminant des rendements en grains du riz dans cette écologie (Akassimadou et al, 2014). La caractérisation texturale a mis en évidence, en avale et dans la partie médiane, l'apparition d'une texture plus fine de type argilo-limoneux résultant du drainage de la partie amont et de l'accumulation des éléments fins et surtout au dynamisme de l'eau dans cette partie. De l'aval vers l'amont il a été enregistré un accroissement du jaunissement et d'une réduction des teintes grises $(2,5 \mathrm{Y})$. Ce qui s'expliquerait par une diminution de la fertilité relativement de l'aval du basfond au fur et à mesure qu'on remonte vers la tête sableuse (Raunet, 1985). Vu la morphologie, la texture fine et le drainage l'installation des cultures devrait se faire en pleine saison des pluies pour contrer la 
sécheresse et il faudra éviter les conditions d'engorgement et de ressuyage qui sont préjudiciables au développement racinaire et à l'alimentation minérale notamment l'alimentation azoté selon Raunet (1985). Dans ce bas-fond les teintes sont grises $(2,5 \mathrm{Y}$ et $5 \mathrm{Y})$ et gleyiques (gley 1) caractéristique des fluvisols. En effet les fluvisols et gleysols sont soumis à la réduction du fer due à l'inondation permanente du milieu (Raunet 1985). Cette coloration gris-noir des horizons de surface $0-20 \mathrm{~cm}$ met en relief la présence de matière organique caractérisé par une large couche humifère dans la fosse du bas-fond et l'absence de fer dans le bas-fond. Cela a été mise en mis en évidence par Barthes (1991) dans le bas-fond de Paracou en Guyane. La dominance de ces teintes laisse croire que la toxicité ferreuse (ADRAO, 2006), l'une des contraintes les plus répandues dans les bas-fonds (ADRAO, 2002; Fageriaet al., 2002) est inexistante ou mineure, contrairement aux sols hydromorphes (gleysols) de la région du Bélier (Zro Bi et al., 2012) qui présentent des risques réels de toxicité ferreuse. L'absence de toxicité ferreuse pourrait s'expliquer aussi par la texture équilibrée dans le bas-fond permettant un bon drainage dans le milieu compte tenu son non confinement et de la texture fine du sol qui offre une bonne aération réduisant alors les phénomènes d'oxydation (ADRAO, 2002), ce qui constitue une action atténuante et de lutte contre le phénomène de la toxicité ferreuse (ADRAO, 2006). Dans cette étude, la

\section{CONCLUSION}

L'étude sur la caractérisation morpho-pédologique et physico-chimiques des sols d'un bas-fond secondaire dans la région de savane guinéenne, a contribuée à une meilleure connaissance et compréhension des phénomènes qui se déroulent dans les sols en submersion. Elle a pu mettre en évidence l'existence de poches sableuses sur les parties latérales, mais également dans la partie médiane, atténuant ainsi le potentiel agronomique et menaçant l'existence même de ce type de bas-fond dans la région. En outre, le $\mathrm{K}$ s'est avéré être l'élément déficient dans ces sols, d'où carte morpho-pédologique montre un rétrécissement de la largeur de l'écologie bas-fond au fur et à mesure qu'on remonte le layon vers l'amont. En effet, la largeur du bas-fond est 170 mètres environ en aval et de 125 $\mathrm{m}$ après $350 \mathrm{~m}$ en remontant le layon. La distribution des sols montre un remblai argileux dominé par les texture argilo-limoneuse, argilo-limono-sableux. Ces résultats coïncident avec les travaux de Raunet (1985) sur la description des bas-fonds en Afrique. En effet, selon lui les bas-fonds se distinguent par un tronçon amont compris entre les têtes sableuses et large de moins de $100 \mathrm{~m}$ et un tronçon aval à remblai argileux entaillé d'un cours d'eau, et large de plus de $100 \mathrm{~m}$. En effet, du point de vu morphologique, on note une largeur du bas-fond de plus 100 mètres environ, avec un sol à texture variable, correspond à un bas-fond secondaire tel que décrit par Raunet (1985). La relative richesse en matière organique des horizons de surface du bas-fond, tel que constatée dans notre étude, pourrait aussi s'expliquer par le phénomène d'hydromorphie, qui bloque l'évolution de la matière organique, lequel va s'accumuler en surface, créant ainsi un sol organique (Lavigne et al, 1996). Cependant, ce processus peut réduire considérablement le $\mathrm{pH}$ et aboutir à la formation de sols très acides à faible $\mathrm{CEC}$. De plus le rapport $\mathrm{C} / \mathrm{N}$ est égal à 10,2 dans le sol de bas-fonds ce qui traduit d'une vitesse de décomposition rapide de la matière organique dans ce sol.

la nécessité d'apporter du $\mathrm{K}$ si l'on veut accroitre les rendements agricoles de manière durable. Toutefois, ces sols présentent des textures équilibrées, permettant un bon drainage et empêchant ainsi les phénomènes de toxicité. On déduit de cette étude que les paysages de sommet cuirassé, démantelé, avec un bas-fond secondaire sur granito-gneiss en zone de savane Guinéenne, offrent un bon potentiel pour la riziculture, alors que l'existence de ce bas-fond est menacée par l'ensablement.

\section{REMERCIEMENTS}

Les Auteurs remercient le département des sciences du sol de l'Université Felix Houphouët-Boigny d'Abidjan, Cocody (Côte d'Ivoire)

\section{BIBLIOGRAPHIE}

ADRAO, 2002. Rapport annuel: points saillants des activités: toxicité ferreuse dans les bas-fonds la rouille du riz, pp. 28-29.
ADRAO, 2006.Toxicité ferreuse dans les systèmes à base riz d'Afrique de l'ouest, Centre du riz 
pour l'Afrique (ADRAO) Cotonou, Bénin. 196 pp. ISBN : 9291133027 (PDF)

Akassimadou EF., Koné B., Yao G. F., Zadi F., Konan F., Traoré M. J. and Yao-Kouamé A. 2014. Rice response to Phosphorus and Potassium in fluvisol of second order lowland in a Guinea savanna zone of Sub-Saharan Africa. International Journal of Plant \&Soil Science 3(3): 232-247, 2014; Article no. IJPSS.2014.002

Albergel j., Lamacherej.m., Lidon b., Gadelle f. , 1993.Mise en valeur agricole des bas-fonds au Sahel. Typologie, fonctionnement hydrologique, potentialités agricoles. Rapport final d'un projet CCE DG12, CIEH, Ouagadougou, $335 \mathrm{p}$.

Andriesse W, Fresco Lo. 1991. A Characterization of Rice-growing Environments in West Africa. AgricEcosys and Environ $1991 ; 33$ : 377-95.

Barthes B. (1991). Influence des caractères pédologiques sur la répartition spatiale de deux espèces du genre EPERUA (CAESALPINIACEAE) en forêt guyanaise (dispositif "Forêt naturelle" du C.T.F.T. à Paracou, Sinnamary, Guyane). ORSTOM, Cayenne $17 \mathrm{p}$.

Berton S. 1988. La maîtrise des crues dans les basfonds. Petits et micro-barrages en Afrique de I'Ouest. Dossier n ${ }^{\circ} 12$ CF/GRET/ACCT. $474 \mathrm{p}$.

Browers M. 1974. Étude morpho-pédologique de reconnaissance pour l'implantation des périmètres irrigués : région de Bagré-Sud. IRAT, Montpellier pp. 5-37. publishing. Royaume Uni. 305 p.

Bremner J.M. 1996. Nitrogen-total. Dans Methods of soil analysis. Part 3 - Chemical Methods. Sparks D.L., Page A.L., Helmke P.A. and Loeppert R.H. (Ed.). American Society of Agronomy, Madison, USA. SSSA Book Series 5: $1085-1122$

CPSC., 1967 :Commission de pédologie et de cartographie des sols. 1967.Classification des sols. Tableaux des classes, sous-classes, groupes et sous-groupes des sols. Service de classification des sols. INRA, France. $96 \mathrm{p}$.

Deville P.L. et Boucher L. 1996. Les Bas-fonds en Afrique Tropicale Humide. Collection le point sur CF/CTA/GRET, $415 \mathrm{p}$.

Fageria N. K., Baligar V. C. and Clark R. B. 2002. Micronutrients in crop production. AgronomyV. 77.267 p.
Jamin J.Y. et Windmeijer P.N.1996. Revue des résultats pour la mise en valeur des bas-fonds en Afrique de l'Ouest. Actes du 4è atelier annuel du Consortium Bas-fonds. ADRAO, Bouaké, 182p.

Killian J. et Teisser. 1973. Méthodes d'investigation pour l'analyse et le classement des bas-fonds dans quelques régions de l'Afrique de l'Ouest: proposition de classification d'aptitudes des terres à la riziculture. Agron. Trop. vol. 28 (2), 156-172.

Koné B., Diatta S., Oikeh S., Gbalou Y., Camara M., Dohm D.D., ASSA A. 2009. Estimation de la fertilité potentielle des ferralsols par la couleur: usage de la couleur en morphopédologie. Canadian Journal of Soil Science, 89 (3) : 331-342.

Kone B., Ettien J B., Amadji G., Diatta S. (2008).Caractérisation de la tolérance de NERICA a la sécheresse de misaison en riziculture pluviale. AfricanCrop Science Journal, Vol. 16. No. 2, June, 2008, pp. 133145

Lavigne Delville Ph., Boucher L. et Vidal L., 1996. "Les bas-fonds en Afrique tropicale humide : stratégies paysannes, contraintes agronomiques et aménagements" in Pichot et al eds. Fertilité $d u$ milieu et stratégies paysannes sous les tropiques humides, actes du séminaire international, CIRAD, pp. 148161

Lidon B., Blanchet F., Legoupil J-C.1996. Le diagnostic rapide hydraulique d'un aménagement de basfond : choix du type, évaluation de l'impact en relation avec son coût. Aménagement et mise en valeur des bas-fonds au Mali. Bilan et perspectives nationales, intérêt pour la zone de savane Ouest-africaine. Actes du séminaire, 21- 25 Octobre 1996, Sikasso, Mali. Colloques, Cirad, Montpellier, France, pp. 191-199.

Mameri C, Mohamed K, Miezan M.K, 2009. Intensification de la riziculture de bas-fonds dans le Sine-Saloum (Sénégal). CahAgric 2008 ; 17 : 451- 5.doi : 10.1684/agr.2008.0205

Nelson D.W. and Sommers L.E., 1996. Total carbon, organic carbon, and organic matter. Dans Methods of soil analysis. Part 3 - Chemical Methods. Sparks D.L., Page A.L., Helmke P.A. and Loeppert R.H. (Ed.).American Society of 
Agronomy, Madison, USA. SSSA Book Series $5: 961-1010$

Olsen S.R. et Sommers L.E., 1982.Phosphorus. In: Page AL, Miller RH, Kenney DR (ed) Methods of Soil Analysis. Part 2. Chemical and Microbiological Properties, Agronomy 9, Second Edition. Madison, Wisconsin, pp. 403427

Ouedraogo M. 1987. Caractéristiques morphostructurales et hydrologiques du bas-fond de Nahirindio (Province de la Bougouriba, Burkina-Faso). Université de Ouagadougou. pp1-35

Ponnamperuma F. N. 1972. The chemistry of submerged soils. AdvanceAgronomy 24, pp.29 $-96$.

Raunet M. 1985."Bas-fonds et riziculture en Afrique; approche structurale et comparative" L'Agronomie Tropicale, 40 (3) : 181-201

Razafindrakoto M.A 2007. Stratégie paysanne améliorée pour l'accroissement de la fertilité du sol et de sa résistance à l'érosion. Actes des JSIRAUF, Hanoi, 6-9 novembre 2007. 6 P.
Thomas G.W. 1996.Soil pH and soil acidity. In Methods of soil analysis. Part 3 - Chemical Methods. Sparks D.L., Page A.L., Helmke P.A. and Loeppert R.H. (Ed.).American Society of Agronomy, Madison, USA. SSSA Book Series 5: $475-490$.

VIZIER J.F., 1983 - Étude des phénomènes d'hydromorphie dans les sols des régions tropicales à saisons contrastées - Dynamique du fer et différenciation des profils. Travaux et Documents de I'ORSTOM, n'165, $294 \mathrm{p}$.

Zeppenfeld T.T et Vlaar J.C.J.1990.Mise en valeur des bas-fonds en Afrique de l'ouest. Synthèse préliminaire de l'état des connaissances. $\mathrm{CIEH}$ Ouagadougou. 149p.

Zro Bi G.F.,Yao-Kouamé A et Kouamé K.F. 2012. Évaluation statistique et spatiale de la fertilité rizicole des sols hydromorphes (gleysols) de la région du Bélier (Côte d'Ivoire). Tropicultura, 30, 4, 236-242. 